\title{
EXTENDED AXION ELECTRODYNAMICS: OPTICAL ACTIVITY INDUCED BY NONSTATIONARY DARK MATTER
}

\author{
A.B. Balakin 1 and N.O. Tarasova 2 \\ Department of General Relativity and Gravitation, Kazan Federal University, \\ Kremlevskaya str., 18, 420008, Kazan, Russia
}

\begin{abstract}
We establish a new self-consistent Einstein-Maxwell-axion model based on the Lagrangian, which is linear in the pseudoscalar (axion) field and its four-gradient and includes the four-vector of macroscopic velocity of the axion system as a whole. We consider extended equations of the axion electrodynamics, modified gravity field equations, and discuss nonstationary effects in the phenomenon of optical activity induced by axions.
\end{abstract}

\section{Introduction}

Axions (pseudo-Goldstone bosons) are considered to be Weakly Interacting Massive Particles (WIMPs) appearing as a result of spontaneous phase transition predicted by Peccei and Quinn [1]. These (hypothetic) particles can play a fundamental role in the formation of Dark Matter, whose contribution into the Universe energy balance is estimated to be about $23 \%$ (see, e.g., [2] - [5]). The axion electrodynamics established by Weinberg and Wilczek [6]-8] gives us a new instrument for the Dark Matter investigation, since the model of coupling between photons and pseudoscalar (axion) field proposed by $\mathrm{Ni}$ in [9] predicts the effect of polarization rotation, when the electromagnetic waves travel through the axion system (see, e.g., [10]-[13]). The optical activity is the best known but not unique phenomenon, which can be induced by axions in the electrodynamic systems. We expect that birefringence, dynamooptical, etc. effects, which are well-known in the classical electrodynamics of moving media, can be found in axion-photons systems as well. Keeping in mind this idea, we intend to establish a number of new models unified terminologically by the common title "Extended Axion Electrodynamics". Non-minimal extension of the EinsteinMaxwell-axion theory [14] was the first step in that direction. In this short note we discuss the class of models, which satisfy the following requirements: first, the

\footnotetext{
${ }^{1}$ e-mail: Alexander.Balakin@ksu.ru

${ }^{2}$ e-mail: anti-neitrino@yandex.ru
} 
electrodynamics is linear; second, the pseudoscalar field equation is of the second order in derivatives; third, the cross-terms in the Lagrangian are linear in the pseudoscalar field and its four-gradient; fourth, the Lagrangian of the model includes the macroscopic velocity four-vector of the system as a whole, but does not contain its derivatives.

\section{Extended model of the axion-photon coupling}

\subsection{On the Lagrangian of the extended model}

The standard Einstein-Maxwell-axion model is based on the Lagrangian formalism with the action functional

$$
\begin{gathered}
S_{(0)}=\int d^{4} x \sqrt{-g} \mathcal{L}_{0} \\
\mathcal{L}_{(0)}=\frac{R+2 \Lambda}{\kappa}+\frac{1}{2} F_{m n} F^{m n}+\frac{1}{2} \phi F_{m n}^{*} F^{m n}+\Psi_{0}^{2}\left[-\nabla_{m} \phi \nabla^{m} \phi+V\left(\phi^{2}\right)\right] .
\end{gathered}
$$

Here, $g$ is the determinant of the metric tensor $g_{i k}, \nabla_{m}$ is the covariant derivative, $R$ is the Ricci scalar, $\kappa \equiv \frac{8 \pi G}{c^{4}}$ is the Einstein coupling constant, $\Lambda$ is the cosmological constant. The Maxwell tensor $F_{m n}$ is given by

$$
F_{m n} \equiv \nabla_{m} A_{n}-\nabla_{n} A_{m}, \quad \nabla_{k} F^{* i k}=0
$$

where $A_{m}$ is an electromagnetic potential four-vector; $F^{* m n} \equiv \frac{1}{2} \epsilon^{m n p q} F_{p q}$ is the tensor dual to $F_{p q} ; \epsilon^{m n p q} \equiv \frac{1}{\sqrt{-g}} E^{m n p q}$ is the Levi-Civita tensor, $E^{m n p q}$ is the absolutely antisymmetric Levi-Civita symbol with $E^{0123}=1$. It is the third term in the Lagrangian that describes the pseudoscalar-photon interaction [9]. The symbol $\phi$ stands for a pseudoscalar field, this quantity being dimensionless. The axion field itself, $\Phi$, is considered to be proportional to this quantity $\Phi=\Psi_{0} \phi$ with a phenomenological constant $\Psi_{0}$. The function $V\left(\phi^{2}\right)$ describes the potential of the pseudoscalar field.

Now we extend the Lagrangian (2) by the terms, which are quadratic in the Maxwell tensor $F_{m n}$, are linear in $\phi$ or in $\nabla_{k} \phi$, and contain the normalized fourvector $U^{k}\left(U^{k} U_{k}=1\right)$. The quantity $U^{k}$ describes the macroscopic velocity of the axion system as a whole, and may be chosen as the time-like eigen-vector of the stress-energy tensor of the pseudoscalar (axion) field. In order to list all the 
irreducible scalars, which satisfy these requirements, let us remind the important identity

$$
F^{i k} F_{k j}^{*}=\frac{1}{4} \delta_{j}^{i} F^{m n} F_{m n}^{*}
$$

Clearly, all the invariants, which we could construct using $g_{i j}, F^{i k}, F_{m n}^{*}, U^{k}$, as well as, $\phi$ or $\nabla_{k} \phi$, definitely contain at least one convolution of the type $F^{i k} F_{k j}^{*}$. Thus, it is easy to check that due to (4) all the new terms in the extended Lagrangian can be reduced to the invariant

$$
\mathcal{L}_{(\mathrm{int})}=\frac{1}{2} \nu F^{m n} F_{m n}^{*} U^{k} \nabla_{k} \phi,
$$

where $\nu$ is some new coupling constant introduced phenomenologically.

\subsection{Extension of the axion electrodynamics}

The variation of the action functional containing the sum of Lagrangians $\mathcal{L}_{(0)}+\mathcal{L}_{(\mathrm{int})}$ with respect to the four-vector potential $A_{i}$ gives the equations of axion electrodynamics

$$
\nabla_{k} H^{i k}=0
$$

Here the excitation tensor $H^{i k}$ is given by the term

$$
H^{i k}=F^{i k}+F^{* i k}(\phi+\nu \mathcal{D} \phi)
$$

and $\mathcal{D}=U^{k} \nabla_{k}$ is the convective derivative. Using the linear constitutive equations

$$
H^{i k}=C^{i k m n} F_{m n}
$$

we readily obtain that the linear response tensor $C^{i k m n}$ now takes the form

$$
C^{i k m n}=\frac{1}{2}\left(g^{i m} g^{k n}-g^{i n} g^{k m}\right)+\frac{1}{2} \epsilon^{i k m n}(\phi+\nu \mathcal{D} \phi) .
$$

This means that the dielectric permittivity and magnetic impermeability tensors of the axion-photon system are the same as in vacuum, i.e.,

$$
\varepsilon^{i m}=2 C^{i k m n} U_{k} U_{n}=\Delta^{i m},
$$

where $\Delta^{i m}=g^{i m}-U^{i} U^{m}$ is the projector, and

$$
\left(\mu^{-1}\right)_{p q}=-\frac{1}{2} \eta_{p i k} C^{i k m n} \eta_{m n q}=\Delta_{p q}
$$

where $\eta_{p i k} \equiv \epsilon_{p i k j} U^{j}$. The tensor of magneto-electric coefficients

$$
\nu_{p}^{m}=\eta_{p i k} C^{i k m n} U_{n}=-\Delta_{p}^{m}(\phi+\nu \mathcal{D} \phi),
$$

describing optical activity effects (see, e.g., [15]) is now characterized by additional term linear in $\mathcal{D} \phi$. 


\subsection{Pseudoscalar field evolution}

Variation of the extended action functional with respect to the pseudoscalar field $\phi$ gives the equation

$$
\nabla_{k} \nabla^{k} \phi+\phi V^{\prime}\left(\phi^{2}\right)=\frac{1}{4 \Psi_{0}^{2}}\left[F_{m n}^{*} F^{m n}(\nu \theta-1)+\nu \mathcal{D}\left(F_{m n}^{*} F^{m n}\right)\right]
$$

where $\theta \equiv \nabla_{k} U^{k}$ is the expansion scalar of the velocity field, and the prime denotes the derivative of the potential $V\left(\phi^{2}\right)$ with respect to the argument.

\subsection{Gravity field equations}

Modified Einstein's equations obtained by the variation of the extended action functional with respect to the metric $g^{p q}$ can be presented in the form

$$
R_{p q}-\frac{1}{2} g_{p q} R=\Lambda g_{p q}+\kappa\left[T_{p q}^{(E M)}+T_{p q}^{(A)}+\nu T_{p q}^{(*)}\right]
$$

Here the stress-energy tensor of the electromagnetic field

$$
T_{p q}^{(E M)}=\frac{1}{4} g_{p q} F_{m n} F^{m n}-F_{p m} F_{q}^{m}
$$

and the stress-energy tensor of the pure axionic field

$$
T_{p q}^{(A)}=\Psi_{0}^{2}\left\{\nabla_{p} \phi \nabla_{q} \phi-\frac{1}{2} g_{p q}\left[\nabla_{m} \phi \nabla^{m} \phi-V\left(\phi^{2}\right)\right]\right\}
$$

are presented by the well-known terms. The tensor

$$
T_{p q}^{(*)}=-\frac{1}{8} F^{m n} F_{m n}^{*}\left(U_{p} \nabla_{q} \phi+U_{q} \nabla_{p} \phi\right)
$$

describes a principally new source-term in the right-hand side of the gravity field equations. Let us mention that the term $\nu T_{p q}^{(*)}$ is obtained by the variation of the term with the interaction Lagrangian (5) by using the formula

$$
\delta U^{i}=\frac{1}{4} \delta g^{p q}\left(U_{p} \delta_{q}^{i}+U_{q} \delta_{p}^{i}\right)
$$

for the variation of the velocity four-vector (see [16] for details). The standard interaction term $\frac{1}{2} \sqrt{-g} \phi F^{m n} F_{m n}^{*}=\frac{1}{4} \phi E^{i k m n} F_{i k} F_{m n}$ does not contribute to the stressenergy tensor in the process of variation with respect to the metric. Thus, the appearance of the term (17) is a new event in the modeling of the gravity field of the photon-axion system. 


\subsection{An example of application}

Let us consider the propagation of test electromagnetic wave coupled to the axionic subsystem of the Dark Matter in the spatially homogeneous FLRW-type spacetime with the scale factor $a(t)$. Let the electromagnetic wave propagate in the direction $0 x$ and be characterized by the potential four-vector $A_{i}=\delta_{i}^{2} A_{2}(t, x)+\delta_{i}^{3} A_{3}(t, x)$. The equations of axion electrodynamics can be now reduced to

$$
\begin{gathered}
{\left[\frac{\partial^{2}}{\partial t^{2}}-\frac{1}{a^{2}} \frac{\partial^{2}}{\partial x^{2}}+H \frac{\partial}{\partial t}\right] A_{2}=-\frac{2 \dot{\Theta}}{a} \frac{\partial}{\partial x} A_{3}} \\
{\left[\frac{\partial^{2}}{\partial t^{2}}-\frac{1}{a^{2}} \frac{\partial^{2}}{\partial x^{2}}+H \frac{\partial}{\partial t}\right] A_{3}=\frac{2 \dot{\Theta}}{a} \frac{\partial}{\partial x} A_{2}}
\end{gathered}
$$

where $H(t)=\frac{\dot{a}}{a}$ is the Hubble function and

$$
\Theta(t) \equiv \frac{1}{2}[\phi(t)+\nu \dot{\phi}(t)]
$$

(in the cosmological context we use the units with $c=1$ ). Clearly, when $\nu=0$ and $\dot{\phi} \neq 0$ the electromagnetic wave can not keep linear polarization, however, in case when $\nu \neq 0$ and the pseudoscalar field evolves exponentially $\phi(t) \propto \exp \left[-\frac{t}{\nu}\right]$, it can be possible. In the approximation of short wavelengths $k>>H$ the solution of (19), (20) for the circularly polarized wave has the form

$$
\begin{gathered}
A_{2}=-A_{0} \sin [W-\varphi(t)], \quad A_{3}=A_{0} \cos [W-\varphi(t)], \\
W=W\left(t_{0}\right)+k\left[\int_{t_{0}}^{t} \frac{d t^{\prime}}{a\left(t^{\prime}\right)}-x\right], \quad \varphi(t) \equiv \Theta(t)-\Theta\left(t_{0}\right),
\end{gathered}
$$

where $k$ is a constant reciprocal to the wavelength. On the one hand, the quantity $\varphi(t)$ is expressed in terms of $\phi$ and $\dot{\phi}$ (see (23) and (21) ) and describes the rotation angle of the polarization vector of the electromagnetic wave traveling through the axion system; it can be studied in optical experiments. On the other hand, it is well-known that in the cosmological context the function $\dot{\phi}$ can be represented in terms of the Dark Matter energy-density $\mathcal{E}$ and pressure $\mathcal{P}$ as follows

$$
\dot{\phi}= \pm \frac{1}{\Psi_{0}} \sqrt{\mathcal{E}(t)+\mathcal{P}(t)} .
$$

Thus, the extended axion electrodynamics can be considered as a tool for investigation of the nonstationary effects in the evolution of the axionic Dark Matter. 
Acknowledgments The authors are grateful to Dr. Zayats A.E. for fruitful comments. This work was supported by the FTP Scientific and ScientificPedagogical Personnel of the Innovative Russia (grants Nos 16.740.11.0185 and 14.740.11.0407), and by the RFBR (grants Nos. 11-02-01162 and 11-05-97518-pcenter-a).

\section{References}

[1] R.D. Peccei and H.R. Quinn, Phys. Rev. Lett., 38, 1440 (1977).

[2] G.G. Raffelt, Phys. Rep., 198, 1 (1990).

[3] M.S. Turner, Phys. Rep., 197, 67 (1990).

[4] A. Del Popolo, Astron. Rep., 51, 169 (2007).

[5] R. Battesti et al, Lect. Notes Phys., 741, 199 (2008).

[6] S. Weinberg, Phys. Rev. Lett., 40, 223 (1978).

[7] F. Wilczek, Phys. Rev. Lett., 40, 279 (1978).

[8] F. Wilczek, Phys. Rev. Lett., 58, 1799 (1987).

[9] W.-T. Ni, Phys. Rev. Lett., 38, 301 (1977).

[10] W.-T. Ni, Prog. Theor. Phys. Suppl., 172, 49 (2008).

[11] E. Zavattini et al (PVLAS Collaboration), Phys. Rev. Lett., 96, 110406 (2006).

[12] S.-J. Chen, H.-H. Mei and W.-T. Ni, Mod. Phys. Lett., A 22, 2815 (2007).

[13] R. Battesti et al, Eur. Phys. J., D 46, 323 (2008).

[14] A.B. Balakin and W.-T. Ni, Class. Quantum Grav., 27, 055003 (2010).

[15] T.H. O'Dell, The electrodynamics of magneto-electric media (Amsterdam: North-Holland, 1970).

[16] A.B. Balakin, Gravitation and Cosmology, 13, 163 (2007). 